\title{
Clinical and molecular surveillance of drug resistant vivax malaria in Myanmar (2009-2016)
}

\author{
Myat Htut Nyunt ${ }^{1,2}$, Jin-Hee Han ${ }^{1}$, Bo Wang ${ }^{3}$, Khin Myo Aye², Kyin Hla Aye², Seong-Kyun Lee ${ }^{1}$, Ye Htut²,
} Myat Phone Kyaw ${ }^{2}$, Kay Thwe Han ${ }^{2 \dagger}$ and Eun-Taek Han ${ }^{1 *+}$

\begin{abstract}
Background: One of the major challenges for control and elimination of malaria is ongoing spread and emergence of drug resistance. While epidemiology and surveillance of the drug resistance in falciparum malaria is being explored globally, there are few studies on drug resistance vivax malaria.

Methods: To assess the spread of drug-resistant vivax malaria in Myanmar, a multisite, prospective, longitudinal study with retrospective analysis of previous therapeutic efficacy studies, was conducted. A total of 906 from nine study sites were included in retrospective analysis and 208 from three study sites in prospective study. Uncomplicated vivax mono-infected patients were recruited and monitored with longitudinal follow-up until day 28 after treatment with chloroquine. Amplification and sequence analysis of molecular markers, such as mutations in pvcrt-O, pvmdr1, pvdhps and pvdhfr, were done in day-0 samples in prospective study.

Results: Clinical failure cases were found only in Kawthaung, southern Myanmar and western Myanmar sites within 2009-2016. Chloroquine resistance markers, pvcrt-O 'AAG' insertion and pvmdr1 mutation (Y976F) showed higher mutant rate in southern and central Myanmar than western site: $66.7,72.7$ vs $48.3 \%$ and $26.7,17.0$ vs $1.7 \%$, respectively. A similar pattern of significantly higher mutant rate of antifolate resistance markers, pvdhps (S382A, K512M, A553G) and pvdhfr (F57L/I, S58R, T61M, S117T/N) were noted.

Conclusions: Although clinical failure rate was low, widespread distribution of chloroquine and antifolate resistance molecular makers alert to the emergence and spread of drug resistance vivax malaria in Myanmar. Proper strategy and action plan to eliminate and contain the resistant strain strengthened together with clinical and molecular surveillance on drug resistance vivax is recommended.
\end{abstract}

Keywords: Malaria, Drug resistance, Plasmodium vivax, Myanmar, Molecular surveillance

\section{Background}

Plasmodium vivax is the most globally widespread malaria parasite, causing significant high public health issues in many countries. World Health Organization (WHO) estimated 13.8 million vivax cases globally in 2015. Although vivax was believed to be a benign

\footnotetext{
*Correspondence: etaekhan@gmail.com

${ }^{\dagger}$ Kay Thwe Han and Eun-Taek Han contributed equally to this work

${ }^{1}$ Department of Medical Environmental Biology and Tropical Medicine,

School of Medicine, Kangwon National University, Chuncheon, Republic of Korea

Full list of author information is available at the end of the article
}

infection, death related to severe vivax malaria was estimated around 1400-14900 cases in 2015 globally [1].

A decreasing trend in malaria promises the possibility of malaria elimination and many endemic countries are moving forward to malaria elimination [1]. One of the major challenges to achieve the elimination goal is drug-resistant falciparum and vivax infection. Unlike falciparum, drug-resistant vivax is difficult to detect, confirm and monitor because of the nature of vivax malaria, such as the presence of hypnotize stage, low level parasitaemia, asymptomatic carriers, and lack of long-term in vitro testing $[2,3]$. 
In the era of pre-elimination, relatively increased prevalence on vivax was observed [1]. In Myanmar, vivax malaria was the second most common malaria species composed of approximately 30 percent of all malaria cases until 2014 [1]. Afterward, the relative prevalence of the vivax has been increasing. Chloroquine (Chloroquine Phosphate Tablet BP, Remedica LtdCyprus) $25 \mathrm{mg} / \mathrm{kg}$ for 3 days followed by primaquine (Remedica Ltd-Cyprus) $0.25 \mathrm{mg} / \mathrm{kg}$ for 14 days is the recommended treatment for vivax malaria in Myanmar while ACT (artemisinin-based combinational therapy) has been using to treat falciparum malaria since 2003 . Although artemisinin resistance falciparum malaria was confirmed by clinically and molecular approaches, very few document on drug resistance vivax malaria was reported in Myanmar.

Chloroquine-resistant vivax malaria was first reported in Papua New Guinea in 1989 [2]. Chloroquine-resistant vivax has been confirmed in ten countries, including Myanmar [1], and treatment failure within day 28 or chemoprophylaxis failure with chloroquine was reported in 21 countries [3]. Unfortunately, there are no accepted and validated molecular markers for chloroquine or other anti-malarial for resistant vivax malaria. However, potential molecular makers for chloroquine-resistant vivax, such as mutations in pvcrt-O (P. vivax chloroquine resistance transporter-O) and pvmdr1 ( $P$. vivax multidrug resistance protein 1$)$ and antifolate resistant vivax such as pvdhps (P. vivax dihydropteroate synthetase) and $p v d h f r$ ( $P$. vivax dihydrofolate reductase) were used for molecular detection to estimate the underlying drug resistance. As there is no documented study on molecular markers analysis on vivax malaria, clinical and molecular markers analysis was conducted in multi-sentinel sites study in Myanmar.

\section{Methods}

\section{Study site and participants}

The study included the retrospective analysis of previously conducted therapeutic efficacy studies (TES) of chloroquine in uncomplicated vivax malaria in Myanmar and prospective multi-site, longitudinal study by clinical and molecular markers analysis. From 2009 to 2012, TES on vivax malaria was conducted in nine sentinel sites (Fig. 1) which covered most of the malaria-endemic areas in Myanmar. In 2012, Shwegyin, in the southern part of central Myanmar, was selected for TES of chloroquine as this study site has a high burden of both falciparum and vivax malaria among migrant goldmine workers. In 2015-2016, TES of chloroquine on vivax malaria was conducted in Buthidaung, in the western border area and Kawthaung, southern Myanmar.

\section{Procedure}

All procedures on case selection, recruitment and followup were carried out according to the standardized protocol recommended by WHO [4]. Briefly, uncomplicated vivax mono-infected patients over 6 years old with fever or history of fever within previous $48 \mathrm{~h}$, were recruited and treated with chloroquine standard dose calculated by body weight, followed by observation with follow-up schedule, i.e., day 3, 7, 21 and 28 after treatment. Clinical and parasitological assessment was done on each followup day. All anti-malarials used in this study were provided by the national malaria control programme.

Blood samples were taken on day 0 and all follow-up days for microscopic examination and molecular analysis. Blood film examination on peripheral blood smear was carried out after 3\% Giemsa stain for $45 \mathrm{~min}$. Blood film examination was done as described [4] and calculated as parasite count per $\mu \mathrm{L}$ of blood. For molecular analysis, finger-prick blood samples were collected on filter paper (Whatman $\odot$ ), dried and stored in plastic bags with desiccant until analysis. Molecular markers analysis was conducted only in prospective study sites such as Kawthaung, Shwegyin and Buthidaung.

\section{Molecular analysis}

All day-0 samples were subjected to analysis for distribution and spread of chloroquine and antifolate drug resistance markers. Filter papers were prepared and extracted for parasite genomic DNA using QIAamp DNA Blood Mini Kit (QIAGEN) according to manufacturer's instruction. The target genes were amplified by using the specific pair of primers (Table 1). In this study, chloroquine resistance marker, 'AAG' insert in pvcrt-O; multidrug resistance marker, mutations in $p v m d r 1$; antifolate resistance makers, mutations in $p v d h p s$ and $p v d h f r$ were amplified and analysed.

PCR reactions were performed in a reaction mixture that contained $0.25 \mathrm{mM}$ of each dNTP, $10 \mathrm{mM}$ Tris- $\mathrm{HCl}$ (pH 9.0), $30 \mathrm{mM} \mathrm{KCl}, 1.5 \mathrm{mM} \mathrm{MgCl}, 1.0$ units of Taq polymerase (Accupower ${ }^{\odot}$ premix, Bioneer, Seoul, Korea), $0.02 \mu \mathrm{M}$ primers, and $2 \mu \mathrm{L}$ of genomic DNA.

For target gene amplifications, initial denaturation at $95{ }^{\circ} \mathrm{C}$ for 10 min was followed by 35 cycles of $95^{\circ} \mathrm{C}$ for $30 \mathrm{~s}, 58{ }^{\circ} \mathrm{C}$ (pvdhps) or $60{ }^{\circ} \mathrm{C}$ (pvcrt-O) or $62{ }^{\circ} \mathrm{C}$ (pvdhps and $p v d h f r$ ) for $45 \mathrm{~s}, 72^{\circ} \mathrm{C}$ for $1 \mathrm{~min}$, and a final extension of $72{ }^{\circ} \mathrm{C}$ for $10 \mathrm{~min}$. Amplified products were checked by $1 \%$ agarose gel electrophoresis stained with Red safe ${ }^{\odot}$ (iNtRON, Seongnam, Republic of Korea). The PCR clean-up was proceeded by MEGAquick-spin DNA fragment purification Kit (iNtRON, Republic of Korea) and sequencing. The nucleotide and amino acid sequences were aligned and analysed by Lasergene ${ }^{\circledR}$ software 


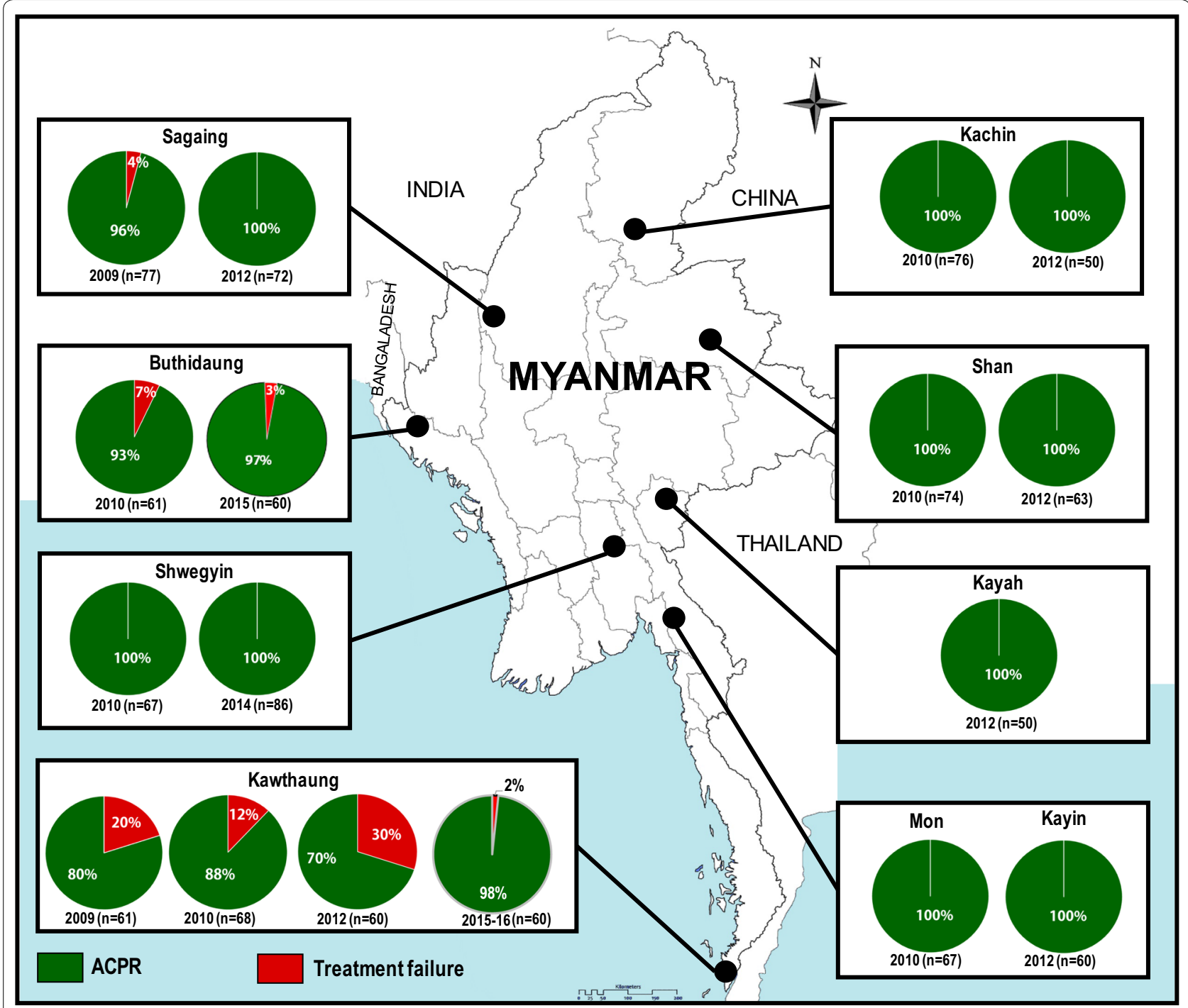

Fig. 1 Retrospective and prospective analysis of the clinical surveillance of drug resistance in vivax malaria in Myanmar (2009-2016). Retrospective analysis was conducted in 906 vivax infected cases in nine sentinel sites. Prospective study with molecular surveillance was conducted in Buthidaung (western Myanmar in 2015), Shwegyin (central Myanmar in 2014) and Kawthaung (southern Myanmar in 2015-2016). Clinical failure cases were found only at southern and western Myanmar

Table 1 Pairs of primers used to amplify the target genes

\begin{tabular}{lll}
\hline Target gene & Primer name & Primer sequences $\left(\mathbf{5}^{\prime} \rightarrow \mathbf{3}^{\prime}\right)$ \\
\hline pvcrt-O & Pvcrto_F & TCC TTG CCG CTG ATT CTA CG \\
& Pvcrto_R & GGT AAC GTT CAT CGG GGG TT \\
pvmdr1 & Pvmdr1_F3 & GGA TAG TCA TGC CCC AGG ATT G \\
& Pvmdr1_R3 & CAT CAA CTT CCC GGC GTA GC \\
pvdhfr & Pvdhfr_F1 & ATG GAG GAC CTT TCA GAT GTA TT \\
& Pvdhfr_R1 & CCA CCTTGC TGT AAA CCA AAA AGT \\
& & CCA GAG \\
pvdhps & Pvdhps_D & GGTTTA TTT GTC GAT CCT GTG \\
& Pvdhps_B & GAG ATT ACC CTA AGG TTG ATG TAT C \\
\hline
\end{tabular}

(DNASTAR, Madison, WI, USA) using the reference strain of Sal-1 retrieved from Plasmodium data base [5]. The nucleotide sequences were submitted to GenBank under accession numbers KX000945-KX000959.

\section{Statistical analysis}

For clinical data, efficacy outcomes were classified as adequate clinical and parasitological response (ACPR) for successful cure cases until day 28 after treatment or treatment failure (TF) that may be early treatment failure (ETF): failure until day 3 after treatment; or late treatment failure (LTF): failure within days 3 to 28. Data were counter-checked and analysed by MS Excel and SPSS 
(Version 22.0. IBM Corp., Armonk, NY, USA). Pearson's Chi squared test or Fisher's exact test was used to determine with a $P$ value of $<0.05$ accepted significant. Mann-Whitney test was calculated for non-parametric analysis on non-normal distribution. Individual as well as co-occurrence of mutations were analysed and compared among different sentinel sites.

\section{Ethical consideration}

Participation in this study was entirely voluntary. Written consent was taken from all participants. This project obtained ethical clearance from the ethical committee of the Department of Medical Research, Republic of the Union of Myanmar (Approval No-52/Ethics, 2012 and 31/Ethics, 2015) and institutional ethical committee of the Kangwon National University, Republic of Korea (KWNUIRB-2016-04-005).

\section{Results}

\section{Retrospective analysis of TES}

During 2009-2012, TES of chloroquine on 906 uncomplicated vivax malaria infected cases was conducted in nine sentinel sites in Myanmar (Fig. 1). LTF cases, according to the WHO definition [4], were reported in Kawthaung (southern Myanmar), Sagaing and Buthidaung (western Myanmar) sites only while there was no treatment failure cases until day 28 follow-up in remaining study sites. The highest failure rate was noted in Kawthaung in 2012 (18/60, 30\%). Kawthaung and Buthidaung site showed failure cases in every study period. There was no early treatment failure case in all study sites.

\section{Prospective longitudinal study}

Kawthaung (southern Myanmar), Shwegyin (central Myanmar) and Buthidaung (western Myanmar) were selected for prospective longitudinal study after treatment with chloroquine regimens. A total of 208 uncomplicated vivax cases were included in this study with mean age of $24.2( \pm 9.03)$ years and geometric mean of the parasite density of 4160 with $95 \%$ CI (3567-4851) (Table 2). There was no clinical failure case in Shwegyin, but one LTF case in Kawthaung at day $28(1 / 60,1.7 \%)$ and two in Buthidaung at days 21 and 28 (2/60, 3.3\%).

\section{Molecular marker analysis}

All day-0 samples collected from three sentinel sites were analysed for molecular markers (Fig. 2; Table 3). More than half of the samples (133/204, 63.9\%) showed K10 'AAG' insertion in chloroquine resistance transporter gene, $p v c r t$-O. Among them, Shwegyin showed the highest mutant rate $(64 / 88,72.7 \%)$ followed by Kawthaung (40/60, 66.7\%) and Buthidaung (29/60, 48.3\%). Similarly, pvmdr1 (Y976F) was found in Kawthaung, Shwegyin and Buthidaung as 16/60, 26.7\%; 15/88, 17.0\% and 1/60, 1.7\%, respectively. Interestingly, significant highest mutant rate of F1076L was observed in Buthidaung (38/60, $63.3 \%)(\mathrm{p}=0.067)$. To estimate the chloroquine resistance status, $p v c r t-O$ (K10 insertion) and pvmdr1 mutations (Y976F and F1076L) were analysed together. Only K10 insertion was lowest in Buthidaung (29/60, 48.3\%) with highest rate of F1076L alone or with K10 insertion together. However, the number of isolates showing K10 insert in $p v c r t-O$ gene as well as both $p v m d r 1$ mutations (Y976F and F1076L) was highest in Kawthaung (11/60, $16.7 \%)$ followed by Shwegyin $(11 / 88,12.5 \%)$ and no isolate in Buthidaung (Tables 3, 4). Similarly, all mutations of pvdhps (S382A, A383G, K512M, A553G) showed the highest in Kawthaung $(17 / 60,28.3 \%)$ followed by Shwegyin $(6 / 88,6.8 \%)$ and Buthidaung $(2 / 60,3.3 \%)$ (Table 3$)$. Moreover, half of the samples showed the wild type pvdhps alleles while no wild type in Kawthaung and one only $(1 / 88,1.1 \%)$ in Shwegyin (Table 4$)$.

Furthermore, mutations in $p v d h f r$ gene were analysed and all mutations, F57L/I, S58R, T61M, and S117T/N, showed the highest mutant rate in Shwegyin $(70 / 88$, $79.5 \%$ ) followed by Kawthaung (45/60, 75.0\%). Wild type alleles were found only in Buthidaung (5/60, 8.3\%). Triple or quadruple mutant alleles of $p v d h p s$ was observed and accounted for more than $50 \%$ of samples in all study sites (Table 4). A combined analysis of all mutations in 208 samples totaled 80 different genotypes (Additional file 1).

\section{Discussion}

This study explores the clinical and molecular pattern of candidate drug resistance markers in Myanmar. Retrospective and prospective analysis on TES of chloroquine on vivax malaria (2009-2016) showed that

Table 2 Basic characteristics of the study participants

\begin{tabular}{|c|c|c|c|c|c|}
\hline Characteristics & All site $(n=208)$ & Kawthaung $(n=60)$ & Shwegyin $(n=88)$ & Buthidaung $(n=60)$ & $P$ value \\
\hline Mean age (SD) & $24.16(9.03)$ & $26.27(10.72)$ & $22.59(7.99)$ & $24.35(8.30)$ & 0.091 \\
\hline$M: F$ & 1.6:1:0 & $9.0: 1.0$ & 4.5:1.0 & 60.0:0.0 & 0.002 \\
\hline Parasite count $(\mathrm{p} / \mu \mathrm{L})$ geometric mean $(95 \% \mathrm{Cl})$ & $4160(3567-4851)$ & $3325(2404-4597)$ & $4795(3866-5948)$ & $4225(3140-5685)$ & 0.520 \\
\hline $\operatorname{ACPR}(n, \%)$ & $205,98.6$ & $59,98.3$ & $88,100.0$ & $58,96.7$ & 0.258 \\
\hline
\end{tabular}

$S D$ standard deviation, $M$ male, $F$ female, $C I$ Confident interval, $A C P R$ adequate clinical and parasitological response 


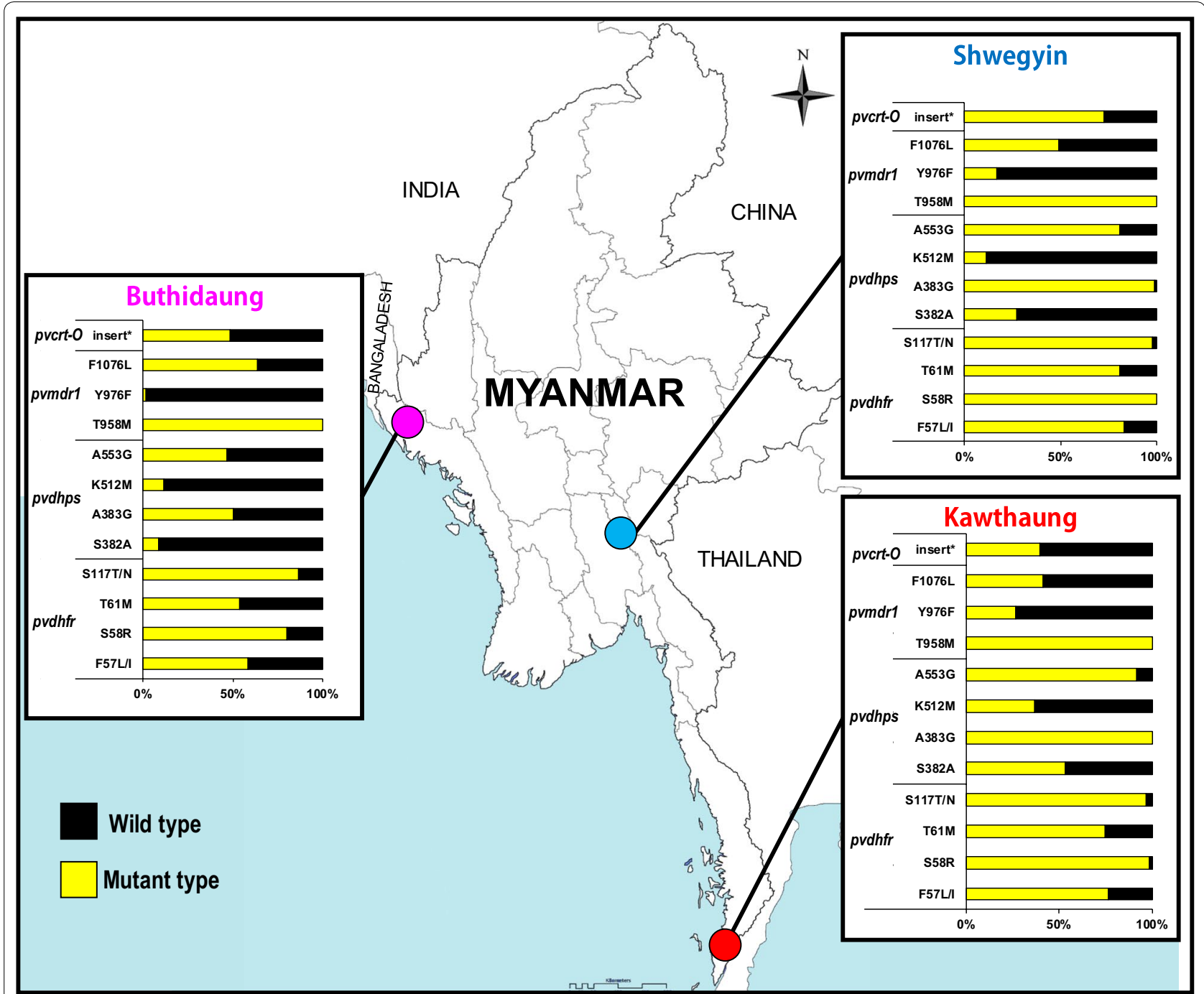

Fig. 2 Molecular marker analysis of drug resistance in vivax malaria in three sentinel sites in Myanmar (2014-2016). A total of 208 from three sentinel sites were included for molecular marker analysis. K10 (AAG) insert of pvcrt-O gene, and mutations of pvmdr1, pvdhps and pvdhfr were shown. Among three sites, the prevalence of all markers except F1076L of pvmdr1 were fewest in Buthidaung site

clinical failures were detected only in southern and western Myanmar. Molecular markers analysis augments additional information on the pattern of drug resistance. In this study, molecular maker analysis indicated widespread distribution of chloroquine, antifolate and multidrug resistance markers, with the highest mutant rate in southern Myanmar.

Surveillance on drug resistance malaria is of great concern for global control and elimination of malaria. As drug-resistant vivax was reported as early as 1980s, its exact global burden is still unknown. Compared to falciparum malaria, drug resistance studies of vivax which focus on epidemiology, drug efficacy and drug resistance mechanism are rare. Drug resistance malaria can be detected by in vivo TES studies, molecular maker analysis, in vitro drug susceptibility testing and drug concentration measurement.

Lack of a standardized culture system limits the usefulness of in vitro susceptibility tests for detection of drug resistance. Although in vivo TES were accepted as a standard method for drug resistance detection, recurrent parasitaemia cases needed to be distinguished between re-infection, recrudescence or relapse. Relapse patterns of vivax malaria also widely differ across geographical regions and no standardized method to exclude relapse or re-infection in TF cases leads to difficulties in interpretation of findings of TES. Currently, clinical surveillance with chloroquine drug level measurement has been accepted to confirm chloroquine-resistant vivax malaria. At least one TF case that showed whole blood 
Table 3 Prevalence of the individual single nucleotide polymorphisms (SNPs) in all study sites

\begin{tabular}{|c|c|c|c|c|c|}
\hline Target genes & Kawthaung $(n=60)$ & Shwegyin $(n=88)$ & Buthidaung $(n=60)$ & All site $(n=208)$ & $P$ value \\
\hline \multicolumn{6}{|l|}{ pvcrt-O } \\
\hline 'AAG'insert & $40(66.7)$ & $64(72.7)$ & $29(48.3)$ & $133(63.9)$ & 0.009 \\
\hline \multicolumn{6}{|l|}{ pvmdr1 } \\
\hline T958M & $60(100.0)$ & $88(100.0)$ & $60(100.0)$ & $208(100.0)$ & $N R$ \\
\hline Y976F & $16(26.7)$ & $15(17.0)$ & $1(1.7)$ & $32(15.4)$ & 0.001 \\
\hline F1076L & $25(41.7)$ & $43(48.9)$ & $38(63.3)$ & $106(51.0)$ & 0.067 \\
\hline \multicolumn{6}{|l|}{ pvdhps } \\
\hline S382A & $32(53.3)$ & $24(27.3)$ & $5(8.3)$ & $61(29.3)$ & 0.000 \\
\hline A383G & $60(100.0)$ & 87 (98.9) & $30(50.0)$ & $177(85.1)$ & 0.000 \\
\hline K512M & $22(36.7)$ & $10(11.4)$ & $7(11.7)$ & 39 (18.8) & 0.000 \\
\hline A553G & $55(91.7)$ & $71(80.7)$ & $28(46.7)$ & $154(74.0)$ & 0.000 \\
\hline \multicolumn{6}{|l|}{ pvdhfr } \\
\hline F57L/l & $46(76.7)$ & $73(83.0)$ & $35(58.3)$ & $154(74.0)$ & 0.001 \\
\hline S58R & $59(98.3)$ & $88(100.0)$ & $48(80.0)$ & $195(93.8)$ & 0.000 \\
\hline $\mathrm{T} 61 \mathrm{M}$ & $45(75.0)$ & $71(80.7)$ & $32(53.3)$ & $148(71.2)$ & 0.001 \\
\hline S117T/N & $58(96.7)$ & $86(97.7)$ & 52 (86.6) & $196(94.2)$ & 0.003 \\
\hline
\end{tabular}

Prevalence of SNPs were shown as 'number (percent)' of respective site. All P value were calculated by Chi square test with $95 \% \mathrm{Cl}$

$N R$ not relevant to be calculated

Table 4 Co-prevalence of single nucleotide polymorphisms (SNPs) in different molecular markers in sentinel sites

\begin{tabular}{|c|c|c|c|c|c|}
\hline \multirow[t]{2}{*}{ Target } & \multirow[t]{2}{*}{ Description $^{a}$} & \multicolumn{4}{|l|}{ No. isolate/total (\%) } \\
\hline & & Kawthaung $(n=60)$ & Shwegyin $(n=88)$ & Buthidaung $(n=60)$ & All sites $(n=208)$ \\
\hline \multirow[t]{2}{*}{ pvert-O } & Wild type & $20(33.3)$ & $24(27.3)$ & $31(51.7)$ & $75(36.1)$ \\
\hline & Mutant (AAG insert) & $40(66.7)$ & $64(72.7)$ & $29(48.3)$ & $133(63.9)$ \\
\hline \multirow[t]{4}{*}{ pvmdrl } & Wild type (T, Y, F) $(958,976,1976)$ & $0(0.0)$ & $0(0.0)$ & $0(0.0)$ & $0(0.0)$ \\
\hline & Single mutant $(\mathbf{M}, Y, F)$ & $35(58.3)$ & $46(52.3)$ & $22(36.7)$ & $103(49.5)$ \\
\hline & Double mutant ( $\mathbf{M}, \mathbf{Y}, \mathbf{L} / \mathbf{I})$ & $9(15.0)$ & $27(30.7)$ & $37(61.7)$ & $73(35.1)$ \\
\hline & Triple mutant $(\mathbf{M}, \mathbf{F}, \mathbf{L})$ & $16(26.7)$ & $15(17.0)$ & $1(1.7)$ & $32(15.4)$ \\
\hline \multirow[t]{9}{*}{ pvdhps } & Wild $(S, A, K, A)(382,383,512,553)$ & $0(0.0)$ & $1(1.1)$ & $30(50.0)$ & $31(14.9)$ \\
\hline & Single mutant $(S, \mathbf{G}, K, A)$ & $5(8.3)$ & $15(17.0)$ & $2(3.3)$ & $22(10.6)$ \\
\hline & Double mutant (S, G, K, G) & $18(30.0)$ & $45(51.1)$ & $18(30.0)$ & $81(38.9)$ \\
\hline & Double mutant $(\mathbf{A}, \mathbf{G}, \mathrm{K}, \mathrm{A})$ & $0(0.0)$ & $1(1.1)$ & $0(0.0)$ & $1(0.5)$ \\
\hline & Triple mutant $(\mathbf{A}, \mathbf{G}, \mathrm{K}, \mathbf{G})$ & $15(25.0)$ & $16(18.2)$ & $3(5.0)$ & $34(16.3)$ \\
\hline & Triple mutant (S, G, E, G) & $0(0.0)$ & $2(2.3)$ & $0(0.0)$ & $2(1.0)$ \\
\hline & Triple mutant $(\mathrm{S}, \mathbf{G}, \mathbf{M}, \mathbf{G})$ & $5(8.3)$ & $1(1.1)$ & $5(8.3)$ & $11(5.3)$ \\
\hline & Quadruple mutant $(\mathbf{A}, \mathbf{G}, \mathbf{M}, \mathbf{G})$ & $17(28.3)$ & $6(6.8)$ & $2(3.3)$ & $25(12.0)$ \\
\hline & Quadruple mutant $(\mathbf{C}, \mathbf{G}, \mathbf{E}, \mathbf{G})$ & $0(0.0)$ & $1(1.1)$ & $0(0.0)$ & $1(0.5)$ \\
\hline \multirow[t]{9}{*}{ pvdhfr } & Wild (F, S, T, S) $(57,58,61,117)$ & $0(0.0)$ & $0(0.0)$ & $5(8.3)$ & $5(2.4)$ \\
\hline & Single mutant $(\mathbf{L}, \mathrm{S}, \mathrm{T}, \mathrm{S})$ & $1(1.7)$ & $0(0.0)$ & $2(3.3)$ & $3(1.4)$ \\
\hline & Single mutant $(F, \mathbf{R}, T, S)$ & $1(1.7)$ & $0(0.0)$ & $0(0.0)$ & $1(0.5)$ \\
\hline & Single mutant $(F, S, T, \mathbf{T} / \mathbf{N})$ & $0(0.0)$ & $0(0.0)$ & $5(8.3)$ & $5(2.4)$ \\
\hline & Double mutant (L, $\mathbf{R}, \mathrm{T}, \mathrm{S})$ & $0(0.0)$ & $2(2.3)$ & $1(1.7)$ & $3(1.4)$ \\
\hline & Double mutant (F, $\mathbf{R}, T, \mathbf{N})$ & $13(21.7)$ & $14(15.9)$ & $15(25.0)$ & $42(20.2)$ \\
\hline & Triple mutant $(\mathbf{L}, \mathbf{R}, \boldsymbol{T}, \mathbf{T})$ & $0(0.0)$ & $1(1.1)$ & $0(0.0)$ & $1(0.5)$ \\
\hline & Triple mutant $(F, \mathbf{R}, \mathbf{M}, \mathbf{T})$ & $0(0.0)$ & $1(1.1)$ & $0(0.0)$ & $1(0.5)$ \\
\hline & Quadruple mutant (L/I, $\mathbf{R}, \mathbf{M}, \mathbf{T} / \mathbf{N})$ & $45(75.0)$ & $70(79.5)$ & $32(53.3)$ & $147(70.7)$ \\
\hline
\end{tabular}

\footnotetext{
a Numbers in parentheses indicate the amino acid position. Mutant amino acids are shown in bold. All sequences were aligned with Sal-1 (P. vivax) reference
} sequences from Plasmodium data base 
concentration of chloroquine plus desethylchloroquine more than $100 \mathrm{ng} / \mathrm{mL}$ was observed in ten countries: Brazil, Ethiopia, Indonesia, Malaysia (Borneo), Myanmar, Papua New Guinea, Peru, the Solomon Islands, and Thailand [6].

Although there are no validated molecular markers for drug-resistant vivax malaria, potential candidates were reported. Most of these candidate markers [7-9] were homologues of falciparum drug resistance makers, such as pvmdr1, pvdhp and pvdhfr. K10 insertion ('AAG' insert) in first exon at tenth position of $p v c r t-O$ was also suggested as a chloroquine resistance marker $[7,10]$; it was observed in Thailand (56-89\%) $[7,10]$ and Myanmar (46\%) [7]. In this study, the highest rate of K10 inset alleles was observed in Shwegyin (central Myanmar) (72.7\%) followed by Kawthaung (southern Myanmar) (66.7\%) and Buthidaung (western Myanmar) (48.3\%) indicating high chloroquine resistance in southern Myanmar, which is similar to the artemisinin resistance status [11]. As the chloroquine is the first line treatment for vivax malaria in Myanmar, K10 insert of $p v c r t-O$ gene was widely distributed in all three study sites.

Y976F mutation of pvmdr1 was found to be associated with reduced susceptibility of chloroquine in Thailand and Indonesia [12] but not in Madagascar [13]. Moreover, Y976F of pvmdr1 gene has been associated with higher susceptibility to artesunate and mefloquine [12]. In this study, Y976F was detected in Kawthaung (26.7\%), Shwegyin (17.0\%) and Buthidaung (1.7\%). Compared to neighbouring countries, Y976F was highest in Cambodia (89\%) [14], followed by Thailand (8-25\%) [10, 14, 15], China-Myanmar border (3\%) [16], and India (0\%) [17]. Another mutation of $p v m d r 1$, F1096L was observed widely but was suggested to be neutral for drug resistance [18]. In combined analysis of $p v c r t-O$ and $p v m d r 1$ mutations, chloroquine resistance markers were widely distributed in all three study sites but with a lower mutant rate in western Myanmar.

Similarly, pvdhps and pvdhfr showed a significant role in antifolate drug-resistant vivax malaria [19]. F57I/L, S58R, T61M, and S117T/N of pvdhfr were found to be associated with pyrimethamine resistance [20] and S382A, S383G, A553G of pvdhps were associated with sulfadoxine resistance [21]. Plasmodium vivax was supposed to have a certain degree of innate resistance to sulfadoxine and S383G and A553G could be responsible inducers for resistance [22]. In Myanmar, relatively high rates of these mutations were noted in southern and central Myanmar.

Double mutations $(\mathrm{S} 58 \mathrm{R}$ and $\mathrm{S} 117 \mathrm{~T} / \mathrm{N})$ or quadruple mutations (F57L/I, S58R, T61M, and S117T) of $p v d-$ $h f r$ gene were found in Thailand (96\%), Myanmar (71\%),
Korea (1\%), Cambodia (94\%), and India (40\%) [23, 24]. In this study, overall quadruple mutations was $147 / 208$ (70.7\%) and contributed more than half of samples in all three study sites, indicating high pyrimethamine resistance in Myanmar. According to $p v d h p s$ and $p v d h f r$ data, antifolate resistance in vivax infection in Myanmar should not be neglected, although sulfadoxine-pyrimethamine is not the drug of choice for vivax malaria.

Within 2009-2016, treatment regimen for vivax malaria was not changed and trend on clinical efficacy of chloroquine is similar except some fluctuations in Kawthaung study site. It is difficult to rule-out the drug resistance factor resulting in treatment failure in this study sites without molecular analysis in previous years. As the drug resistance alone is not responsible for treatment failure [4], clinical response and prevalence of molecular markers is not similar in this study.

\section{Conclusions}

This study is the first multisite, clinical and molecular surveillance of drug-resistant vivax malaria in Myanmar, exploring the neglected niche of the infection. According to current anti-malarial treatment guidelines in Myanmar, chloroquine is the first-line treatment for vivax malaria. Most of the study sites showed 100\% ACPR except in the southern and western Myanmar sites. Wide distribution of chloroquine and antifolate resistance molecular markers revealed the spread of the drug-resistant parasite population in Myanmar. High mutant rates of most of the vivax drug resistance molecular markers in southern and central Myanmar were similar to that of artemisinin resistance falciparum malaria, indicating higher anti-malarial resistance burden of falciparum and vivax in southern Myanmar. An appropriate strategy and action plan to contain or eliminate drug-resistant vivax malaria in Myanmar is recommended.

\section{Additional file}

Additional file 1. Genotypes based on the co-prevalence of the drug resistance vivax makers.

\footnotetext{
Authors' contributions

MHN, YH, KTH, MPK, and ETH conceived and designed the study; KTH, KMA and KHA collected field samples and field data management; $\mathrm{MHN}, J \mathrm{HH}$, BW, and SKL performed the laboratory experiments and data analysis, MHN and ETH wrote the manuscript. All authors read and approved the final manuscript.

\section{Author details}

${ }^{1}$ Department of Medical Environmental Biology and Tropical Medicine, School of Medicine, Kangwon National University, Chuncheon, Republic of Korea.

${ }^{2}$ Department of Medical Research, Yangon, Myanmar. ${ }^{3}$ Department of Clinical Laboratory, The First Affiliated Hospital of Anhui Medical University, Anhui,

China.
} 


\section{Acknowledgements}

We thank the staff from Parasitology Research Division and local health authorities from Kawthaung, Shwegyin and Buthidaung for their help with collection of the field samples. The authors thank the malaria patients actively involved in this study.

\section{Competing interests}

The authors declare that they have no competing interests.

\section{Availability of data and materials}

The datasets analysed in this study are available from the research team but restrictions may apply to the availability of data, which were used under license of the current study, and so are not publicly available. However, data are available from the corresponding authors upon reasonable request and with the permission of the original research team

\section{Ethical approval and consent to participants}

Participation in this study was entirely voluntary. Written consent was taken from all participants. This project obtained ethical clearance from the ethical committee of the Department of Medical Research, Republic of the Union of Myanmar (Approval No-52/Ethics, 2012 and 31/Ethics, 2015) and institutional ethical committee of the Kangwon National University, Republic of Korea (KWNUIRB-2016-04-005)

\section{Funding}

This study was supported by the US Agency for International DevelopmentPresident's Malaria Initiative (USAID-PMI) through WHO Umbrella Grant for intensifying Anti-malarial Drug Resistance Monitoring and Response in the Greater Mekong Sub-region and the Korea International Cooperation Agency (KOICA), and the National Research Foundation of Korea (NRF2014R1A2A1A11052079), Ministry of Science, ICT and Future Planning Republic of Korea.

Received: 17 January 2017 Accepted: 8 March 2017

Published online: 16 March 2017

\section{References}

1. WHO. World Malaria Report 2015. Geneva: World Health Organization; 2015.

2. Rieckmann $\mathrm{KH}$, Davis DR, Hutton DC. Plasmodium vivax resistance to chloroquine? Lancet. 1989;334:1183-4.

3. WHO. Control and elimination of Plasmodium vivax malaria: a technical brief. Geneva: World Health Organization; 2015

4. WHO. Methods for surveillance of antimalarial drug efficacy. Geneva: World Health Organization; 2009.

5. PlasmoDB_-Plasmodium genomics resource. [http://plasmodb.org]. Accessed 2 Mar 2017

6. WHO. Global report on antimalarial drug efficacy and drug resistance: 2000-2010. Geneva: World Health Organization; 2010.

7. Lu F, Lim CS, Nam D-H, Kim K, Lin K, Kim T-S, et al. Genetic polymorphism in pvmdr1 and pvcrt-o genes in relation to in vitro drug susceptibility of Plasmodium vivax isolates from malaria-endemic countries. Acta Trop. 2011:117:69-75.

8. Lu F, Wang B, Cao J, Sattabongkot J, Zhou H, Zhu G, et al. Prevalence of drug resistance-associated gene mutations in Plasmodium vivax in centra China. Korean J Parasitol. 2012;50:379-84.

9. Lekweiry KM, Boukhary AOMS, Gaillard T, Wurtz N, Bogreau H, Hafid JE, et al. Molecular surveillance of drug-resistant Plasmodium vivax using pvdhfr, pvdhps and pvmdr1 markers in Nouakchott, Mauritania. J Antimicrob Chemother. 2012;67:367-74.

10. Suwanarusk R, Russell B, Chavchich M, Chalfein F, Kenangalem E, Kosaisavee $V$, et al. Chloroquine resistant Plasmodium vivax: in vitro characterisation and association with molecular polymorphisms. PLOS ONE. 2007;2:e1089.

11. WHO. Strategic framework for artemisinin resistance containment in Myanmar (MARC) 2011-2015. Yangon: WHO country office for Myanmar; 2011.
12. Suwanarusk R, Chavchich M, Russell B, Jaidee A, Chalfein F, Barends M, et al. Amplification of pvmdr1 associated with multidrug-resistant Plasmodium vivax. J Infect Dis. 2008;198:1558-64.

13. Barnadas C, Ratsimbasoa A, Tichit M, Bouchier C, Jahevitra M, Picot S, et al. Plasmodium vivax resistance to chloroquine in Madagascar: clinica efficacy and polymorphisms in pvmdr1 and pvcrt-o Genes. Antimicrob Agent Chemother. 2008:52:4233-40.

14. Lin JT, Patel JC, Kharabora O, Sattabongkot J, Muth S, Ubalee R. Plasmodium vivax isolates from Cambodia and Thailand show high genetic complexity and distinct patterns of $P$. vivax multidrug resistance gene 1 (pvmdr1) polymorphisms. Am J Trop Med Hyg. 2013;88:1116-23.

15. Rungsihirunrat K, Muhamad P, Chaijaroenkul W, Kuesap J, Na-Bangchang K. Plasmodium vivax drug resistance genes; pvmdr 1 and pvcrt-o polymorphisms in relation to chloroquine sensitivity from a malaria endemic area of Thailand. Korean J Parasitol. 2015;53:43-9.

16. Feng J, Zhou D, Lin Y, Xiao H, Yan H, Xia Z. Amplification of pfmdr 1, pfcrt, pvmdr1, and K13 propeller polymorphisms associated with Plasmodium falciparum and Plasmodium vivax isolates from the China-Myanmar border. Antimicrob Agent Chemother. 2015;59:2554-9.

17. Ganguly S, Saha P, Guha SK, Das S, Bera DK, Biswas A, et al. In vivo therapeutic efficacy of chloroquine alone or in combination with primaquine against vivax malaria in Kolkata, West Bengal, India, and polymorphism in pvmdr1 and pvcrt-o genes. Antimicrob Agent Chemother. 2013;57:1246-51.

18. Imwong M, Pukrittayakamee S, Pongtavornpinyo W, Nakeesathit S, Nair S,

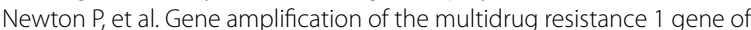
Plasmodium vivax isolates from Thailand, Laos, and Myanmar. Antimicrob Agent Chemother. 2008;52:2657-9.

19. Price RN, Auburn S, Marfurt J, Cheng Q. Phenotypic and genotypic characterisation of drug-resistant Plasmodium vivax. Trend Parasitol. 2012;28:522-9.

20. Marfurt J, Monbrison F, Brega S, Barbollat L, Müller I, Sie A, et al. Molecular markers of in vivo Plasmodium vivax resistance to amodiaquine plus sulfadoxine-pyrimethamine: mutations in pvdhfr and pvmdr1. J Infect Dis 2008;198:409-17.

21. Imwong M, Pukrittayakamee S, Cheng Q, Moore C, Looareesuwan $\mathrm{S}$, Snounou $\mathrm{G}$, et al. Limited polymorphism in the dihydropteroate synthetase gene (dhps) of Plasmodium vivax Isolates from Thailand. Antimicrob Agent Chemother. 2005;49:4393-5.

22. Korsinczky M, Fischer K, Chen N, Baker J, Rieckmann K, Cheng Q. Sulfadoxine resistance in Plasmodium vivax is associated with a specific amino acid in dihydropteroate synthase at the putative sulfadoxine-binding site. Antimicrob Agent Chemother. 2004;48:2214-22.

23. Lu F, Lim CS, Nam DH, Kim K, Lin K, Kim T-S, et al. Mutations in the antifolate-resistance-associated genes dihydrofolate reductase and dihydropteroate synthase in Plasmodium vivax isolates from malaria-endemic countries. Am J Trop Med Hyg. 2010;83:474-9.

24. Saralamba N, Nakeesathit S, Mayxay M, Newton PN, Osorio L, Kim J-R, et al. Geographic distribution of amino acid mutations in DHFR and DHPS in Plasmodium vivax isolates from Lao PDR, India and Colombia. Malar J. 2016;15:484.

\section{Submit your next manuscript to BioMed Central and we will help you at every step:}

- We accept pre-submission inquiries

- Our selector tool helps you to find the most relevant journal

- We provide round the clock customer support

- Convenient online submission

- Thorough peer review

- Inclusion in PubMed and all major indexing services

- Maximum visibility for your research

Submit your manuscript at www.biomedcentral com/submit 